\title{
How to make a bilayer exciton condensate flow
}

\author{
Jung-Jung Su and A.H. MacDonald \\ Department of Physics, The University of Texas at Austin, Austin, TX 78712, USA
}

\begin{abstract}
Bose condensation is responsible for many of the most spectacular effects in physics because it can promote quantum behavior from the microscopic to the macroscopic world. Bose condensates can be distinguished by the condensing object; electron-electron Cooper-pairs are responsible for superconductivity, Helium atoms for superfluidity, and ultracold alkali atoms in vapors for coherent matter waves. Electron-hole pair (exciton) condensation has maintained special interest because it has been difficult to realize experimentally, and because exciton phase coherence is never [1] perfectly spontaneous. Although ideal condensates can support[2] an exciton supercurrent, it has not been clear 3] how such a current could be induced or detected, or how its experimental manifestation would be altered by the phase-fixing exciton creation and annihilation processes which are inevitably present. In this article we explain how to induce an exciton supercurrent in separately contacted bilayer condensates, and predict electrical effects which enable unambiguous detection.
\end{abstract}

PACS numbers:

The order parameter of an exciton condensate is

$\Psi(\vec{r})=|\Psi(\vec{r})| \exp (i \phi(\vec{r}))=\left\langle\hat{\psi}_{e}^{\dagger}(\vec{r}) \hat{\psi}_{h}(\vec{r})\right\rangle=\rho(h, \vec{r} ; e, \vec{r})$

where $\hat{\psi}^{\dagger}$ and $\hat{\psi}$ are electron creation and annihilation operators, $\phi(\vec{r})$ is the condensate phase, the labels $e$ (electron) and $h$ (hole) refer to the states between which phase coherence is established (nearly!) spontaneously, and $\rho(h, \vec{r}, e, \vec{r})$ is the anomalous density matrix. Microscopic considerations suggest [4] that spontaneous coherence is likely between a conduction band with occupied states inside a Fermi surface and a valence band with occupied states outside a nearly [5] identical Fermi surface. Part of the reason that exciton condensation has not been easy to realize is that sufficiently perfect nesting between conduction and valence bands is unlikely to occur naturally. The systems of interest here are artificially fabricated bilayers in which the electrons and holes are in well separated two-dimensional electron systems(2DESs), either semiconductor quantum wells [6, 7] or graphene layers 8 separated by a dielectric barrier. The dielectric barrier reduces the strength of exciton creation and annihilation processes, and gate control of the density in each layer allows electron and hole band Fermi surfaces to be tuned to the same area. Although simple to describe, this quantum engineering is difficult [6, 7, 9] to execute successfully. Exciton condensation has so far been realized 10 only in the quantum Hall regime in which band dispersion is irrelevant allowing spontaneous coherence to occur between spatially separated conduction bands, or spatially separated valence bands, under circumstances that are achieved routinely. The considerations explained in this article apply to quantum Hall exciton condensates in the Corbino geometry [11, 12], in which current flows across the 2DES bulk, but not directly to the Hall bar geometry [13] in which currents flows along the 2DES edge.

In their pioneering work on exciton Bose condensation Blatt et al. [3] argued that because an exciton is neutral, condensation can not lead to spectacular electrical effects. Experimental studies of quantum Hall exciton condensates have already made it clear [10, 14] that this pessimistic conclusion is not valid. The key technical capability not anticipated in 1962 is the possibility of making independent electrical contact 15] to the electron and hole parts of the condensate. In this article we explain how condensation leads to a reorganization of the low-energy charged fermion degrees of freedom which is responsible for dramatic changes in the transport properties of separately contacted condensed bilayers.

Spectacular electrical effects in exciton condensates are enabled by the possibility of exciton superflow. The key issue which arises in addressing these phenomena theoretically is understanding how a supercurrent of neutral excitons can be driven by electrochemical potential differences. We consider a bilayer with contacts to both left $(\mathrm{L})$ and right $(\mathrm{R})$ ends of the separate quantum wells (top $(\mathrm{T}=\mathrm{e})$ and bottom $(\mathrm{B}=\mathrm{h}))$ between which coherence is established, as illustrated schematically in Fig.[1]. (In the case of the Corbino geometry transport of quantum Hall condensates $\mathrm{L}$ and $\mathrm{R}$ refer to the outer and inner edges of an annular 2DES.) For the sake of definiteness we focus our attention on voltage biased transport; our analysis is easily extended to the case of current biased transport and to systems with additional voltage probes along the sample length. The observables in this transport geometry are the currents and voltages in the leads. Exciton condensation alters transport properties by introducing an anomalous symmetry-breaking field in the fermion quasiparticle Hamiltonian (more technically in the one-particle Greens function) which enforces interlayer phase coherence, opens up a gap at the Fermi level, and allows charge to move freely between layers. In mean-field-theory, the quasiparticle amplitude for tunneling between top $(\mathrm{T})$ and bottom $(\mathrm{B})$ layers is:

$$
\left\langle T, \overrightarrow{r^{\prime}}\left|H_{H F}\right| B, \vec{r}\right\rangle=V_{c}\left(\left|\overrightarrow{r^{\prime}}-\vec{r}\right|\right) \rho\left(T, \overrightarrow{r^{\prime}} ; B, \vec{r}\right)
$$

where $V_{c}(r)=e^{2} / \epsilon \sqrt{r^{2}+d^{2}}$ is the inter-layer Coulomb interaction and $\rho\left(T, \overrightarrow{r^{\prime}} ; B, \vec{r}\right)$ is the inter-layer component of the density matrix. $\left(\rho\left(T, \overrightarrow{r^{\prime}} ; B, \vec{r}\right)\right.$ is non-zero 

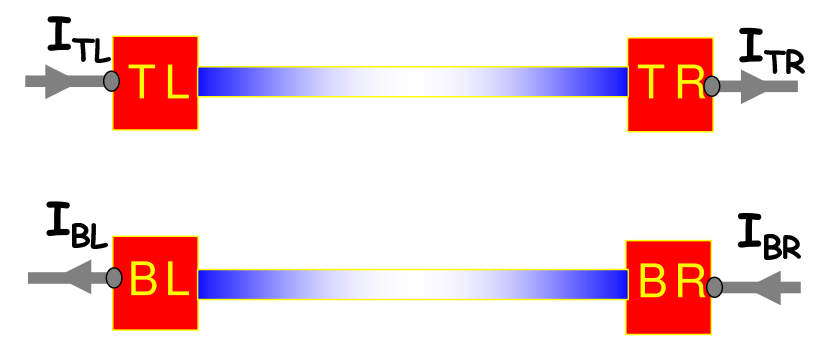

FIG. 1: Schematic illustration of a separately contacted bilayer exciton condensate. $L$ and $R$ represen 1 the left and right ends of a quantum well system or the outes and inner edges of an annular quantum Hall bilayer conden. sate. Currents through the four leads can be tuned externally by varying the three independent voltage differences. Ou convention for positive currents is indicated by the arrows at. tached to the leads. Transport properties are simplest wher there is a gap (indicated by white fill in this schematic illus. tration) in the bulk.

only in the condensed state.) These quasiparticle tunneling terms are present even when the microscopic elec. tron Hamiltonian does not include inter-layer transfer processes. In a mean-field theory microscopic particlenumber conservation in each layer is recovered when the exciton pair potential is calculated self-consistently; inter-layer quasiparticle currents are then precisely compensated by exciton condensate supercurrents. Steady state exciton supercurrents are possible, but only when the lead voltages are chosen to satisfy a condition that we explain below. Our main predictions are qualitative in nature. The quantitative properties of a particular exciton condensate system will depend on the details of its Hamiltonian, including its disorder potentials. We illustrate some of our ideas on transport in exciton condensates below by considering a one-dimensional toy model with short-range inter-layer interactions, using the NEGF[16] method to evaluate steady-state nonequilibrium density-matrices.

When $\rho(T, \vec{r} ; B, \vec{r})$ has a spatial phase gradient, the quasiparticle Hamiltonian supports [2] non-zero currents even when the system is in equilibrium, i.e. when all four chemical potentials are identical. Because the exciton condensate cannot carry charge, the condensate current (CC) has only 2] a counterflow component, i.e.

$$
j_{T}^{C C}(x)+j_{B}^{C C}(x)=0 .
$$

The total current in each layer can be expressed as the sum of the condensate current and a quasiparticle current $(\mathrm{QC})$ driven by lead voltage differences. The $\mathrm{QC}$ conduction is related via the Landauer-Büttiker formula to the transmission coefficients of quasiparticle waves incident from the various leads. In order to drive steady state condensate current, the gate voltages must be chosen so that the counter-flow component of the $\mathrm{QC}$ is not

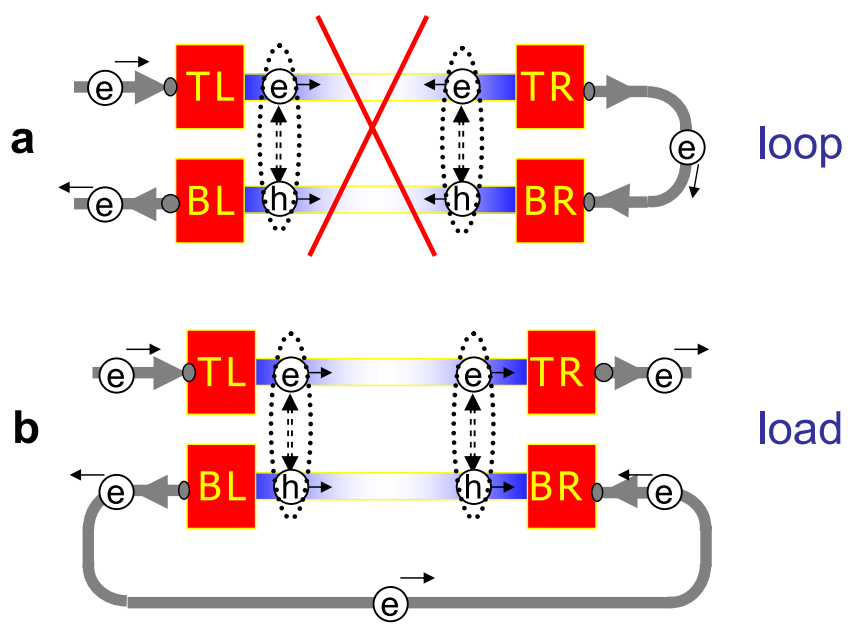

FIG. 2: Loop and load geometries. Fig. $\mathbf{a}$ and $\mathbf{b}$ represent respectively loop and load geometries. For bilayer exciton condensates only the load geometry $\mathbf{b}$ permits steady state exciton superflow.

spatially constant:

$$
\left[\partial_{x}\left(j_{T}^{C C}(x)-j_{B}^{C C}(x)\right)\right]=-\left[\partial_{x}\left(j_{T}^{Q C}(x)-j_{B}^{Q C}(x)\right)\right]
$$

Condensate currents are induced by space-dependent quasiparticle counterflow currents. This relationship between quasiparticle $(\mathrm{QC})$ and condensate $(\mathrm{CC})$ counterflow currents follows from the separate conservation of charge in each layer. Since condensate currents cannot enter the leads, they must be present in the bulk when across the sample $I_{T L}+I_{B L} \neq 0$ or $I_{T R}+I_{B R} \neq 0$. (Here we use the conventions of Fig. [ 1] for the lead current signs.) For every electron that is reflected from the top layer to the bottom layer at the left edge of the sample, an exciton must be launched. It follows that $\left(I_{T L}+I_{B L}\right)$ is the exciton supercurrent emitted from the left side of the sample and that $\left(I_{T R}+I_{B R}\right)$ is the exciton supercurrent absorbed on the right. Steady state condensate currents are possible only when these two quantities are equal:

$$
I_{T L}+I_{B L}=I_{T R}+I_{B R} .
$$

In the linear response regime, the four lead currents are proportional to the three independent electrochemical potential differences. Eq. (4) places a restriction on the two independent difference ratios.

One convenient way to reduce the lead-voltage-space dimension experimentally is to connect two contacts with a resistor $R$, for example in either the loop geometry or the load geometry illustrated in Fig.( 2). In the load geometry, the contact voltages are limited to the surface on which $I_{B L}=I_{B R}=\left(V_{B L}-V_{B R}\right) / R$. The consequences of this resistive link between contacts are easily anticipated in the most common limit in which the gap due to condensation is large enough or disorder strong enough to prevent quasiparticle current conduction across the 
sample. Bulk quasiparticle currents are expected to be negligible for Corbino geometry transport in the quantum Hall regime, and we anticipate that they will also be negligible in zero-field bilayer exciton condensates when these are ultimately realized. When quasiparticles cannot flow across the sample, $I_{T L}=I_{B L}$ and $I_{T R}=I_{B R}$. It follows that Eq. (5) is always satisfied in the load geometry. Because it is possible to induce an exciton supercurrent in the bulk of the bilayer, a large charge current can flow through the circuit with a resistance due only to the load resistor and contact resistances at the two ends of the sample. In the case of quantum Hall bilayers, we predict a Corbino resistance for the load geometry which remains constant as temperature $T \rightarrow 0$ on bilayer quantum Hall plateaus which are due to exciton condensation [14]. This behavior is in stark contrast with the dramatically increasing resistance expected at low temperatures on plateaus not associated with exciton condensation.

Although the loop geometry of Fig.(2) is naively compatible with steady state counterflow currents, we predict that it cannot support steady state exciton superflow because it does not guarantee that the the exciton supercurrent emitted at the left end of the sample is identical to that absorbed at the right end. Indeed for $I_{T R}>0$ the loop geometry will lead to $V_{T R}>V_{B R}$ and therefore to quasiparticle current flowing in the wrong direction from top to bottom.

In the load geometry, the effective two-probe quasiparticle conductances at left and right $G_{L, R}=\left(e^{2} / h\right) T_{L, R}$ depend on the details of these contacts and on the quasiparticle Hamiltonian near the sample ends. Taking the $T R$ lead as ground, the voltages on the other leads in this circumstance are $V_{T L}=I\left(\left[T_{L}^{-1}+T_{R}^{-1}\right]\left(h / e^{2}\right)+R\right)$, $V_{B L}=I\left(R+T_{R}^{-1}\left(h / e^{2}\right)\right)$, and $V_{B R}=I\left(h / e^{2}\right) T_{R}^{-1}$ where $I_{T L}=I_{B L}=I_{B R}=I_{T R}=I$. When the load resistor $R$ is small, the current which flows through the bilayer systems is limited only by the quantum contact resistances $\left(h / e^{2}\right) T_{L, R}^{-1}$. Even when the microscopic electrons tunneling amplitude is zero, quasiparticles move freely between layers allowing charge to be conducted across the highly resistive bulk by exploiting the parallel load resistor channel.

In Fig.( 3) we plot numerical results for the selfconsistently calculated quasiparticle and condensate current distributions in a one-dimensional tight-bindingmodel bilayer system with an electron band in the top layer and an equal density hole band in the bottom layer. The inter-layer tunneling amplitude in the quasiparticle Hamiltonian of this model is

$$
\left\langle T, I^{\prime}\left|H_{H F}\right| B, I\right\rangle=\delta_{I^{\prime}, I} V \rho\left(T, I^{\prime} ; B, I\right)
$$

where $V$ is the short-range inter-layer interaction strength and $I, I^{\prime}$ is the site indices. Because of the energy gap induced in the quasiparticle Hamiltonian by this tunneling term, the quasiparticle current is deflected to flow between $\mathrm{T}$ and $\mathrm{B}$ leads at both left and right. The numerical results in Fig.( 3) have been obtained
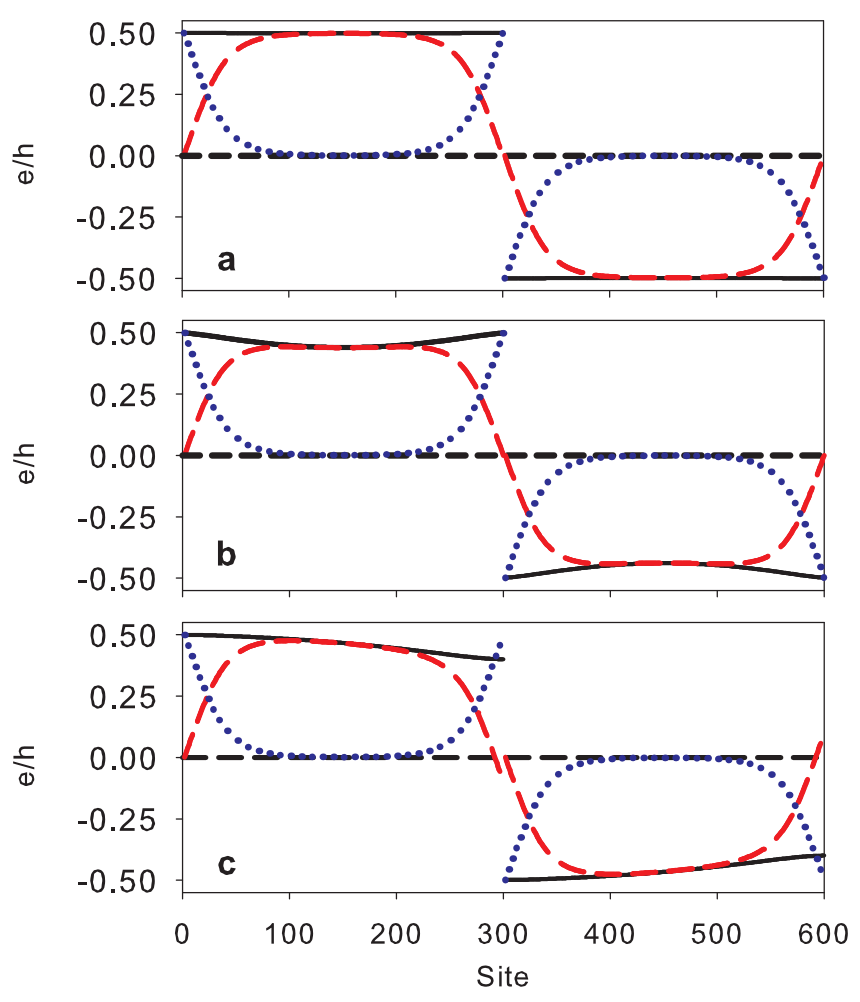

FIG. 3: 1D Toy model self-consistent current distributions. Sites 1 through 300 label the top layer left to right while sites 301 through 600 labels the bottom layer. The site separation is 0.08 Bohr radii. The black solid lines, red dashed lines, and blue dotted lines represent the total current, the condensate current (CC) and the quasiparticle current respectively. In a, $V_{T L}=1.0, V_{T R}=-0.5, V_{B L}=V_{B R}=0.0$ and the bare interlayer tunneling amplitude $\Delta_{t}=0.0$. (Voltages are in Rydberg/e) The total (CC+QC) current is constant and the QP current is very small near the system center. $\mathbf{b}, V_{T L}=0.5, V_{T R}=-0.5, V_{B L}=0.0, V_{B R}=0.0, \Delta_{t}=0.005$. When bare tunneling occurs the total current is no longer a constant, but the transport characteristics in loop geometry do not change in a qualitative way. $\mathbf{c}, V_{T L}=0.5, V_{T R}=$ $-0.5, V_{B L}=0.0, V_{B R}=-0.1, \Delta_{t}=0.005$. Steady state solutions with net exciton creation rates are possible because of bare electron interlayer tunneling. The unit of voltage is Rydberg/e.

by using mean-field theory (Eq.( 6) ) to calculate the quasiparticle Hamiltonian given the density matrix, and the NEGF formalism [16] to evaluate the non-equilibrium density matrices given the lead voltages and the quasiparticle Hamiltonian. In the top panel of Fig.( 3) ,$V_{B(L, R)}=\left(\left(V_{T L}+V_{T R}\right) \pm V^{\prime}\right) / 2$. Because the left and right sides of our model system are identical this choice produces $I_{B L}=I_{B R}$, corresponding to the load geometry. ( $V^{\prime}$ is related to the load wire resistance by $V^{\prime} /\left(V_{T L}-V_{T R}\right)=R /\left(R+T_{L}^{-1}+T_{R}^{-1}\right)$.) When the non-equilibrium mean-field calculation is carried to selfconsistency, the phase of the order parameter develops a spatial gradient and the system carries a steady state 
condensate current.

The total current is constant in each layer as required by charge conservation. In the ordered state, the lowenergy charged degrees of freedom are reorganized from bare electrons which cannot transfer between layers to exciton-condensate quasiparticles which occupy the twolayers simultaneously and coherently. This profound reorganization of low-lying charged excitations decreases the resistance exponentially compared to the case in which the gap in the quasiparticle spectrum is not due to exciton condensation. In the quantum Hall case for example, we predict load geometry resistances that are orders of magnitude smaller at $\nu=1$ (the exciton condensation case) than at $\nu=2$. For the loop geometry, no self-consistent steady-state solutions of the nonequilibrium self-consistent field equations exist. In this case we anticipate enhanced noise due to order-parameter time dependence and dramatically higher steady state time-averaged resistance.

As emphasized in a fundamental early paper by Kohn and Sherrington[1], phase coherence between different bands in a solid can never be completely spontaneous. In the case of bilayer condensates, inter-layer electron tunneling, which creates or annihilates excitons, is expected to be the dominant process which fixes a preferred phase and violates separate charge conservation in each layer. As illustrated in panel b in Fig.( 3), however, weak inter-layer electron tunneling has little effect on transport properties. Most experimental anomalies associated with bilayer exciton condensation require only that the quasiparticle tunneling amplitude be dramatically enhanced compared to its bare values. As we now explain the main consequence of bare-electron inter-layer tunneling is partial relaxation of the current-conservation condition, Eq. (51).

The role of a bare electron inter-layer tunneling amplitude is most simply discussed using the minimal field theory model [2] of a bilayer exciton condensate:

$$
E=\int d r\left[\frac{\hbar^{2} \rho_{s}}{2 m^{*}}|\nabla \theta|^{2}-M \Delta_{t} \cos \theta\right]
$$

where $\theta$ is the inter-layer coherence phase, $\Delta_{t}$ is the bareelectron tunneling amplitude, , $m^{*}$ is the effective mass in the Landau-Ginzburg type theory, $M=2|\rho(T, B)|$ is the condensate order parameter, and $\rho_{s}$ is its superfluid density. In this description $j=\left(\hbar \rho_{s} \partial_{x} \theta\right) / m^{*}$ is the exciton condensate supercurrent. Minimizing this energy functional leads to a sine-Gordon equation from which it follows that

$$
\partial_{x}\left[\frac{1}{2} \lambda^{2}\left(\partial_{x} \theta\right)^{2}+\cos \theta\right]=0 .
$$

where $\lambda^{2}=\left(\rho_{s} / M\right)\left(\hbar^{2} / m^{*} \Delta_{t}\right)$ is the model's Josephson length. From the constant-of-motion in square brackets in Eq.( 8), we obtain an explicitly expression for the difference between the condensates currents at opposite ends of the sample:

$$
j_{R}^{2}-j_{L}^{2}=\left(\cos \theta_{L}-\cos \theta_{R}\right) \frac{2}{\lambda^{2}}\left(\frac{\hbar \rho_{s}}{m^{*}}\right)^{2}
$$

Defining $J \equiv\left(j_{R}+j_{L}\right) / 2$ and $\delta j \equiv j_{R}-j_{L}$, we conclude that steady state collective currents are possible provided that the exciton currents injected at left and absorbed at right differ by less than

$$
\delta j_{\max } \leq \Delta_{t} \frac{2 \rho_{s} M}{m^{*}} \frac{1}{J} .
$$

The limit on $\delta j$ is closely analogous to the critical current predicted [13] for inter-layer tunneling. For contact resistances are $\sim h / e^{2}$ we predict that large conductances will occur when $V_{B R}$ is varied by less than $\sim(h / e) \delta j_{\max }$ from its load resistance value. In our one-dimensional toy model for example $\left(j_{L}=I_{T L} / e, j_{R}=I_{T R} / e\right)$, Eq. (10) implies that $\delta j_{\max } \sim 0.1($ Rydberg $/ h)$

for $\Delta_{t}=0.005$ (Rydberg) and total current $j=$ $0.5(\mathrm{Rydberg} / h)$. Indeed, our NEGF calculations find a self-consistent solution, illustrated in Fig.( 3 c), for the voltage configuration $V_{T L}=0.5, V_{B L}=0.0, V_{T R}=$ $-0.5, V_{B R}=-0.1$ (in (Rydberg/e) units) (corresponding to $\delta j \sim 0.1($ Rydberg $/ h)$ ) but not for larger $\delta j$ in qualitative agreement with this prediction.

This work has been supported by the Welch Foundation and by the National Science Foundation under grant DMR-0606489. AHM acknowledges long-standing stimulating interactions with Jim Eisenstein, Werner Dietche, and Klaus von Klitzing which have informed this analysis and helpful discussions with Mike Lilly, Lars Tiemann, and Ian Spielman.
[1] Kohn, W. \& Sherrington, D. Two kinds of bosons and bose condensates. Rev. Mod. Phys. 42, 1-11 (1970).

[2] Moon, K. et al. Spontaneous inter-layer coherence in double-layer quantum Hall systems: Charged vortices and Kosterlitz-Thouless phase transitions. Phys. Rev. B 51, 5138-5170 (1995).

[3] Blatt, J. M. Böer, K. W. \& Brandt, W. Bose-Einstein condensation of excitons. Phys. Rev. 126, 1691 (1962).

[4] Keldysh, L. V. \& Kozlov, A,N. Collective properties of excitons in semiconductors. Sov. Phys. JETP 27, 521-528 (1968).

[5] Conti, S., Vignale, G. \& MacDonald, A. H. Engineering superfluidity in electron-hole double layers. Phys. Rev. B 57, R6846-R6849 (1998).

[6] Seamons, J. A. Tibbetts, D. R., Reno, J. L. \& Lilly, M. P. Undoped electron-hole bilayers in a GaAsAlGaAs double quantum well. Appl. Phys. Lett., 90, 052103, (2007).

[7] Gupta, K. Das et al., Selective breakdown of Quantum 
Hall edge states and non-monotonic coulomb drag in a GaAs-AlGaAs electron-hole bilayer. Physica E, to appear (2008).

[8] Geim, A. K. \& MacDonald, A. H. Graphene: Exploring carbon flatland. Physics Today 60, 35-41 (2007).

[9] The increase in electrostatic potential between electron and hole layers required to engineer an exciton condensate is qualitatively smaller for graphene layers because they are gapless. At least in this sense, the requirements for engineering exciton condensation are less severe for graphene layers then for semiconductor quantum wells.

[10] Spielman, I.B., Eisenstein, J.P., Pfeiffer, L.N., \& West K.W., Resonantly Enhanced Tunneling in a Double Layer Quantum Hall Ferromagnet Phys. Rev. Lett. 84, 5808 (2000); Phys. Rev. Lett. 87, 036803 (2001).

[11] Tiemann, L. et al., Investigating the transport properties of the excitonic state in quasi-Corbino electron bilayers, Physica E, in press. doi:10.1016/j.physe.2007.09.148
(2008)

[12] Tiemann, L. et al., Critical tunneling currents in the regime of bilayer excitons, New Journal of Physics, to appear (2008).

[13] Rossi, E. Nunez, A. S., \& MacDonald, A. H. Inter-layer transport in bilayer quantum Hall systems. Phys. Rev. Lett. 95, 266804 (2005)

[14] Eisenstein, J. P. \& MacDonald, A. H. Bose-Einstein condensation of excitons in bilayer electron systems. Nature 432, 691-694 (2004).

[15] Eisenstein, J.P., Pfeiffer L.N., \& West K.W., Independently contacted 2-Dimensional Electron Systems in Double Quantum-Wells, Appl. Phys. Lett. 57, 2324 (1990).

[16] Datta, S. Electronic Transport in Mesoscopic Systems, (Cambridge University Press, 1995). 